\title{
Reciprocity laws for generalized higher dimensional Dedekind sums
}

by

\author{
Robin Chapman (Sydney, N.S.W.)
}

We define a class of generalized Dedekind sums and prove a family of reciprocity laws for them. These sums and laws generalize those of Zagier [6]. The method is based on that of Solomon [5].

1. Dedekind sums. For a real number $t$ let $\lfloor t\rfloor$ denote its integer part, i.e., the unique integer with $\lfloor t\rfloor \leq t<\lfloor t\rfloor+1$, and $\{t\}=t-\lfloor t\rfloor$ denote its fractional part. Define

$$
B(t)= \begin{cases}\{t\}-1 / 2 & \text { if } t \notin \mathbb{Z} \\ 0 & \text { if } t \in \mathbb{Z} .\end{cases}
$$

For integers $a, b$ and $c$ with $c>0$ we define the Dedekind sum

$$
s(a, b ; c)=\sum_{j=0}^{c-1} B(a j / c) B(b j / c) .
$$

This is a slight generalization of the usual Dedekind sum which is defined by $s(h, k)=s(1, h ; k)$. The Dedekind sum has various formal properties which we shall not list; one is $s(a, b ; c)=s(a d, b d ; c)$ for $d$ coprime to $c$. If $a$ is coprime to $c$ this shows that $s(a, b ; c)$ equals the classical Dedekind sum $s\left(a^{\prime} b, c\right)$ where $a a^{\prime} \equiv 1(\bmod c)$. Rademacher [4] proved a three-term reciprocity law for these sums.

TheOREM 1. Let $a, b$ and $c$ be pairwise coprime positive integers. Then

$$
s(a, b ; c)+s(b, c ; a)+s(c, a ; b)=-\frac{1}{4}+\frac{1}{12}\left(\frac{a}{b c}+\frac{b}{c a}+\frac{c}{a b}\right) .
$$

The special case of $c=1$ gives the classical reciprocity law:

$$
s(a, b)+s(b, a)=-\frac{1}{4}+\frac{1}{12}\left(\frac{a}{b}+\frac{b}{a}+\frac{1}{a b}\right) .
$$

2000 Mathematics Subject Classification: Primary 11F20. 
Zagier [6] generalized this by considering sums of the form

$$
\sum_{\substack{0 \leq i_{1}, \ldots, i_{n}<a \\ a \mid\left(b_{1} i_{1}+\ldots+b_{n} i_{n}\right)}} B\left(i_{1} / a\right) \ldots B\left(i_{n} / a\right)
$$

where the integers $b_{1}, \ldots, b_{n}$ are each coprime to the positive integer $a$. Zagier proves an $(n+1)$-term reciprocity law for these sums.

We generalize Zagier's formula further to sums of the form

$$
s_{r_{1}, \ldots, r_{n}}\left(a ; b_{1}, \ldots, b_{n} ; \lambda_{1}, \ldots, \lambda_{n}\right)
$$

where the $\widetilde{B}_{r}$ are Bernoulli functions as defined in the next section, $a$ is a positive integer, $b_{1}, \ldots, b_{n}$ are integers coprime to $a, r_{1}, \ldots, r_{n}$ are nonnegative integers and $\lambda_{1}, \ldots, \lambda_{n}$ are real numbers. The case $r_{1}=\ldots=r_{n}=$ 1 and $\lambda_{1}=\ldots=\lambda_{n}=0$ gives the sums studied by Zagier. The classical and Rademacher's Dedekind sums are given by

$$
s(h, k)=s_{1,1}(k ; h,-1 ; 0,0)
$$

and

$$
s(a, b ; c)=s_{1,1}(c ; b,-a ; 0,0) .
$$

2. Bernoulli functions. The Bernoulli polynomials $B_{n}(t)$ are defined by the generating function

$$
\frac{x e^{t x}}{e^{x}-1}=\sum_{n=0}^{\infty} B_{n}(t) \frac{x^{n}}{n !} .
$$

Each $B_{n}(t)$ is a monic polynomial of degree $n$. The first few examples are

$$
B_{0}(t)=1, \quad B_{1}(t)=t-1 / 2, \quad B_{2}(t)=t^{2}-t+1 / 6 .
$$

The Bernoulli numbers are defined by $B_{n}=B_{n}(0)$. For $n \neq 1$ we also have $B_{n}(1)=B_{n}$ since

$$
\sum_{n=0}^{\infty}\left(B_{n}(1)-B_{n}\right) \frac{x^{n}}{n !}=\frac{x e^{x}-x}{e^{x}-1}=x .
$$

On the other hand, $B_{1}=-1 / 2$ but $B_{1}(1)=1 / 2$. We define periodic versions of the Bernoulli polynomials, the Bernoulli functions, by

$$
\widetilde{B}_{n}(t)= \begin{cases}B_{n}(\{t\}) & \text { if } n \neq 1 \text { or } t \notin \mathbb{Z} \\ 0 & \text { if } n=1 \text { and } t \in \mathbb{Z} .\end{cases}
$$


Each $\widetilde{B}_{n}(t)$ has period 1 , and all are continuous save for $\widetilde{B}_{1}(t)$ which equals $B(t)$ in our established notation. Let

$$
\Phi(t, x)=\sum_{n=0}^{\infty} \widetilde{B}_{n}(t) \frac{x^{n-1}}{n !}
$$

denote the generating function of the Bernoulli functions. We have

$$
\Phi(t, x)= \begin{cases}\frac{e^{\{t\} x}}{e^{x}-1} & \text { if } t \notin \mathbb{Z}, \\ \frac{e^{x}+1}{2\left(e^{x}-1\right)} & \text { if } t \in \mathbb{Z} .\end{cases}
$$

It follows that $\Phi(-t, x)=-\Phi(t,-x)$ and so

$$
\widetilde{B}_{n}(-t)=(-1)^{n} \widetilde{B}_{n}(t) .
$$

For positive integers $m$ we easily prove the distribution relation for these generating functions

$$
\sum_{j=0}^{m-1} \Phi((t+j) / m, x)=\Phi(t, x / m) .
$$

As it is apparent that both sides have period 1 in $t$ we may assume that $0 \leq t<1$ and then the identity becomes just the sum of a finite geometric series. An immediate corollary is the distribution relation for the Bernoulli functions:

$$
\sum_{j=0}^{m-1} \widetilde{B}_{n}((t+j) / m)=m^{1-n} \widetilde{B}_{n}(t) .
$$

We would like to expand these expressions as formal power series and equate both sides, for instance for $0<t<1$ we should have

$$
\sum_{n=0}^{\infty} \widetilde{B}_{n}(t) \frac{x^{n}}{n !}=\frac{x e^{t x}}{e^{x}-1}=-\sum_{j=0}^{\infty} x e^{(t+j) x}=-\sum_{j=0}^{\infty} \sum_{k=0}^{\infty}(t+j)^{k} \frac{x^{k+1}}{k !} .
$$

However there seems to be no meaningful way that this gives an identity of power series in $x$. If we were to invert the order of summation, then the coefficients of the $x^{k+1}$ would be non-convergent series. Also we have the embarrassment that the infinite sum of series without a constant term is equated to a series with a non-zero constant term. Nonetheless in the next section we shall see how one can assign a power series expansion to certain infinite sums of exponential series.

3. Formal power series of quotient type. Let $K$ be a field and $G$ be a torsion-free abelian group with the group operation written multiplicatively. Let $K[G]$ denote the group ring. Then the elements of $K[G]$ 
are finite sums $\sum_{g \in G} a_{g} g$ where $a_{g} \in K$ and $a_{g}=0$ with only finitely many exceptions. Indeed $K[G]$ is an integral domain. To see this we may assume that $K[G]$ is finitely generated, in which case it is isomorphic to $K\left[x_{1}, x_{1}^{-1}, x_{2}, x_{2}^{-1}, \ldots, x_{n}, x_{n}^{-1}\right]$ for some $n$. This is clearly an integral domain.

We now define $K\{G\}$ to be the set of all formal infinite linear combinations $\sum_{g \in G} a_{g} g$ where $a_{g} \in K$ and we do not assume that all but finitely many are non-zero. The set $K\{G\}$ contains the group ring $K[G]$ but is not itself a ring in a natural way, but $K\{G\}$ is a vector space over $K$. However we can multiply elements of $K[G]$ and $K\{G\}$ together to get elements of $K\{G\}$ and so $K\{G\}$ is a $K[G]$-module. We say that $f \in K\{G\}$ is of quotient type if there is a non-zero $g \in K[G]$ with $g f \in K[G]$. We denote the set of all $f \in K\{G\}$ which are of quotient type by $K\{G\}_{q}$.

As an example let $G$ be cyclic with generator $x$. Then $f=\sum_{n=-\infty}^{\infty} x^{n} \in$ $K\{G\}$ is of quotient type since $(x-1) f=0$. Our aim is to identify $f \in$ $K\{G\}_{q}$ with $h / g$ whenever $g f=h$ and $g, h \in K[G]$ with $g$ non-zero. This example shows that on some occasions a non-zero formal sum should be identified with zero.

Lemma 1. The set $K\{G\}_{q}$ is a $K[G]$-submodule of $K\{G\}$ containing $K[G]$.

Proof. For $f \in K[G], 1 f=f \in K[G]$ so that $K[G] \subseteq K\{G\}_{q}$.

If $f \in K\{G\}_{q}$ take a non-zero $h \in K[G]$ with $h f \in K[G]$. Then for $g \in K[G]$ we have $h(g f)=g(h f) \in K[G]$ so that $h f \in K\{G\}_{q}$. If $f_{1}$, $f_{2} \in K\{G\}_{q}$ let $h_{1}, h_{2} \in K[G]$ be non-zero with $h_{1} f_{1}, h_{2} f_{2} \in K[G]$. Then $h_{1} h_{2} \neq 0$ and $h_{1} h_{2}\left(f_{1}+f_{2}\right)=h_{2}\left(h_{1} f_{1}\right)+h_{1}\left(h_{2} f_{2}\right) \in K[G]$.

Let $L$ be a field containing $K$ and $\varphi: K[G] \rightarrow L$ be an injective $K$ homomorphism. For instance $\varphi$ could be the inclusion of $K[G]$ in its quotient field. We wish to extend $\varphi$ to a "homomorphism" of $K\{G\}_{q}$ to $L$. (We put homomorphism in quotes since there is no obvious ring structure on $K\{G\}_{q}$.)

Lemma 2. The map $\psi: K\{G\}_{q} \rightarrow L$ given by $\psi(f)=\varphi(g f) / \varphi(g)$ whenever $g, g f \in K[G]$ and $g \neq 0$ is well defined. Also $\psi$ is additive and $\psi(h f)=\varphi(h) \psi(f)$ for $f \in K\{G\}_{q}$ and $h \in K[G]$.

P r o o f. Suppose that $g_{1}$ and $g_{2}$ are non-zero elements of $K[G]$ with $g_{1} f$, $g_{2} f \in K[G]$. Then $g_{2}\left(g_{1} f\right)=g_{1}\left(g_{2} f\right)$ and so $\varphi\left(g_{2}\right) \varphi\left(g_{1} f\right)=\varphi\left(g_{1}\right) \varphi\left(g_{2} f\right)$. As $\varphi$ is injective then $\varphi\left(g_{1}\right), \varphi\left(g_{2}\right) \neq 0$ and so $\varphi\left(g_{1} f\right) / \varphi\left(g_{1}\right)=\varphi\left(g_{2} f\right) / \varphi\left(g_{2}\right)$ so that $\psi$ is well defined.

For $f_{1}, f_{2} \in K\{G\}_{q}$ let $g_{1}, g_{2} \in K[G]$ be non-zero with $f_{1} g_{1}, f_{2} g_{2} \in$ $K[G]$. Then $g_{1} g_{2}\left(f_{1}+f_{2}\right) \in K[G]$ and so 


$$
\begin{aligned}
\psi\left(f_{1}+f_{2}\right) & =\frac{\varphi\left(g_{1} g_{2}\left(f_{1}+f_{2}\right)\right)}{\varphi\left(g_{1} g_{2}\right)}=\frac{\varphi\left(g_{2}\right) \varphi\left(g_{1} f_{1}\right)+\varphi\left(g_{1}\right) \varphi\left(g_{2} f_{2}\right)}{\varphi\left(g_{1}\right) \varphi\left(g_{2}\right)} \\
& =\frac{\varphi\left(g_{1} f_{1}\right)}{\varphi\left(g_{1}\right)}+\frac{\varphi\left(g_{2} f_{2}\right)}{\varphi\left(g_{2}\right)}=\psi\left(f_{1}\right)+\psi\left(f_{2}\right) .
\end{aligned}
$$

Finally let $f \in K\{G\}_{q}$ and $h \in K[G]$ and let $g$ be a non-zero element of $K[G]$ with $g f \in K[G]$. Then $g(h f)=h(g f) \in K[G]$ and so

$$
\psi(h f)=\frac{\varphi(g h f)}{\varphi(g)}=\frac{\varphi(h) \varphi(g f)}{\varphi(g)}=\varphi(h) \psi(f)
$$

4. Exponential sums over lattice points. From now on we fix a positive integer $n$ and take indeterminates $X_{1}, \ldots, X_{n}$. We identify the real Euclidean space $\mathbb{R}^{n}$ with the set of linear forms in $X_{1}, \ldots, X_{n}$ so that we equate a vector $x=\left(x_{1}, \ldots, x_{n}\right)$ with $x_{1} X_{1}+\ldots+x_{n} X_{n}$.

Now let $G$ be the group of all formal power series $\exp \left(x_{1} X_{1}+\ldots+\right.$ $\left.x_{n} X_{n}\right)=e^{x}$ where $x=\left(x_{1}, \ldots, x_{n}\right) \in \mathbb{R}^{n}$. Then $G$ is a torsion free abelian group.

We let $K$ be any field of characteristic zero, so that $G \subseteq K\left[\left[X_{1}, \ldots, X_{n}\right]\right]$, the field of formal power series in $n$ variables. We let $L=K\left(\left(X_{1}, \ldots, X_{n}\right)\right)$ be its quotient field. The inclusion $G \subseteq L$ gives rise to a homomorphism $\varphi: K[G] \rightarrow L$ and so also a map $\psi: K\{G\}_{q} \rightarrow L$. Define, for real $t$,

$$
\omega(t)= \begin{cases}1 & \text { if } t>0, \\ 1 / 2 & \text { if } t=0, \\ 0 & \text { if } t<0 .\end{cases}
$$

Note that $\omega(t)+\omega(-t)=1$. Set

$$
\phi(\lambda, X)=-\sum_{t \in \lambda+\mathbb{Z}} \omega(t) e^{t X} .
$$

Then

$$
\psi(\phi(\lambda, v))=\Phi(\lambda, v)
$$

whenever $v \in \mathbb{R}^{n}$, identifying, as always, $\mathbb{R}^{n}$ with the space of linear forms in $X_{1}, \ldots, X_{n}$.

Let $a_{0}, \ldots, a_{n}$ be pairwise coprime positive integers.

Lemma 3. The group $\mathbb{Z}^{n}$ has elements $u_{0}, \ldots, u_{n}$ with the property that the $u_{j}$ generate $\mathbb{Z}^{n}$ as an additive group and $\sum_{j=0}^{n} a_{j} u_{j}=0$.

Pro of. Let $u_{1}^{\prime}, \ldots, u_{n}^{\prime}$ be $n$ linearly independent vectors in $\mathbb{Q}^{n}$. Set $u_{0}^{\prime}=$ $-a_{0}^{-1} \sum_{j=1}^{n} a_{j} u_{j}^{\prime}$. The abelian group $\Lambda$ generated by the $u_{j}^{\prime}$ is free abelian of rank $n$, and is isomorphic to $\mathbb{Z}^{n}$. If $\psi: \Lambda \rightarrow \mathbb{Z}^{n}$ is an isomorphism, then the $u_{j}$ defined by $u_{j}=\psi\left(u_{j}^{\prime}\right)$ have the desired properties. 
Note that each size $n$ subset of $u_{0}, \ldots, u_{n}$ forms a basis of $\mathbb{R}^{n}$. Also since the $u_{j}$ span $\mathbb{Z}^{n}$ a vector $x \in \mathbb{R}^{n}$ lies in $\mathbb{Z}^{n}$ if and only if all the $u_{j} \cdot x$ are in $\mathbb{Z}$.

For distinct integers $j, k \in\{0, \ldots, n\}$ there is $v_{j k} \in \mathbb{R}^{n}$ such that $u_{i} \cdot v_{j k}$ $=0$ for $i \notin\{j, k\}$ and $u_{j} \cdot v_{j k}=a_{k}$. It follows that $u_{k} \cdot v_{j k}=-a_{j}$. Hence each $v_{j k}$ is in $\mathbb{Z}^{n}$. As $u_{i} \cdot\left(v_{j k}+v_{k j}\right)=0$ for all $i$ we have $v_{j k}+v_{k j}=0$. Similarly $a_{i} v_{j k}+a_{j} v_{k i}+a_{k} v_{i j}=0$.

For a fixed $k$ consider the set $\left\{v_{j k}: j \neq k\right\}$. For $i \neq j$ we have $u_{i} \cdot v_{j k}$ $=a_{k}$ if $i=k$ and $u_{i} \cdot v_{j k}=0$ otherwise. It follows that the set $\left\{v_{j k}: j \neq k\right\}$ is a basis of $\mathbb{R}^{n}$. Also if

$$
x=\sum_{j \neq k} \lambda_{j} v_{j k}
$$

then $u_{j} \cdot x=a_{k} \lambda_{j}$. The vector $x$ lies in $\mathbb{Z}^{n}$ if and only if $u_{j} \cdot x \in \mathbb{Z}$ for each $j$. Taking $j \neq k$ this means that $\lambda_{j}=h_{j} / a_{k}$ where $h_{j} \in \mathbb{Z}$ and taking $j=k$ it means in addition that $\sum_{j \neq k} a_{j} h_{j} \equiv 0\left(\bmod a_{k}\right)$.

We now wish to consider sums of exponentials over regions defined by inequalities such as $u_{j} \cdot z \geq 0$. Let

$$
S_{k}(x)=\sum_{y \in x+\mathbb{Z}^{n}} e^{y} \prod_{\substack{j=0 \\ j \neq k}}^{n} \omega\left(u_{j} \cdot y\right)
$$

Essentially this is the sum of $e^{y}$ over all $y \in x+\mathbb{Z}^{n}$ which lie in the region defined by the inequalities $u_{j} \cdot y \geq 0$ but where each term $e^{y}$ is weighted according to the number of boundary hyperplanes that $y$ lies in. It is crucial that each of these sums is of quotient type.

Proposition 1. Let $0 \leq k \leq n$. Then $S_{k}(x)$ is of quotient type; in fact $\psi\left(S_{k}(x)\right)$

$$
=(-1)^{n} \sum_{\substack{0 \leq i_{0}, \ldots, i_{k-1}, i_{k+1}, \ldots, i_{n}<a_{k} \\ a_{k} \mid\left(a_{0} i_{0}+\ldots+a_{k-1} i_{k-1}+a_{k+1} i_{k+1}+\ldots+a_{n} i_{n}\right)}} \prod_{\substack{j=0 \\ j \neq k}}^{n} \Phi\left(\left(i_{j}+\lambda_{j}\right) / a_{k}, v_{j k}\right)
$$

where $\lambda_{j}=u_{j} \cdot x$.

Proof. For $0 \leq j \leq n$ and $j \neq k$ the vectors $v_{j k}$ lie in $\mathbb{Z}^{n}$ and are linearly independent over $\mathbb{Q}$. Let $\Lambda_{k}$ denote the lattice they generate. Then $\Lambda_{k}$ has finite index in $\mathbb{Z}^{n}$. We shall determine the cosets of $\Lambda_{k}$ in $\mathbb{Z}^{n}$ and split up the sum $S_{k}$ into sums over each coset.

We have seen that

$$
y=\sum_{\substack{j=0 \\ j \neq k}}^{n} \mu_{j} v_{j k}
$$


lies in $\mathbb{Z}^{n}$ if and only if $\mu_{j}=i_{j} / a_{k}$ where $i_{j} \in \mathbb{Z}$ and $\sum_{j=0, j \neq k}^{n} a_{j} i_{j}$ is divisible by $a_{k}$. Thus each coset of $\Lambda_{k}$ in $\mathbb{Z}^{n}$ has a unique representative $y=a_{k}^{-1} \sum_{j} i_{j} v_{j k}$ with $i_{j} \in \mathbb{Z}, 0 \leq i_{j}<a_{k}$, and $a_{k} \mid\left(a_{1} i_{1}+\ldots+a_{k-1} i_{k-1}+\right.$ $\left.a_{k+1} i_{k+1}+\ldots+a_{n} i_{n}\right)$.

Given such a $y$, consider the sum

$$
S_{k}(x, y)=\sum_{z \in x+y+\Lambda_{k}} e^{z} \prod_{\substack{j=0 \\ j \neq k}}^{n} \omega\left(u_{j} \cdot z\right) .
$$

Each $z$ in $x+y+\Lambda_{k}$ has the form

$$
a_{k}^{-1} \sum_{\substack{j=0 \\ j \neq k}}^{n}\left(i_{j}+\lambda_{j}+c_{j} a_{k}\right) v_{j k}
$$

where the $c_{j}$ are arbitrary integers. Then $\omega(u \cdot z)$ depends only on the sign of $i_{j}+\lambda_{j}+c_{j} a_{k}$. We get

$$
S_{k}(x, y)=\sum_{\substack{c_{j} \in \mathbb{Z} \\ j \neq k}} \prod_{\substack{j=0 \\ j \neq k}}^{n} \omega\left(i_{j}+\lambda_{j}+c_{j} a_{k}\right) \exp \left(a_{k}^{-1}\left(i_{j}+\lambda_{j}+c_{j} a_{k}\right) v_{j k}\right) .
$$

It is clear that this is of quotient type and that

$$
\psi\left(S_{k}(x, y)\right)=(-1)^{n} \prod_{\substack{j=0 \\ j \neq k}}^{n} \Phi\left(\left(i_{j}+\lambda_{j}\right) / a_{k}, v_{j k}\right) .
$$

Adding up $S_{k}(x, y)$ over all the coset representatives $y$ gives the stated formula.

Expanding each $\Phi\left(\left(i_{j}+\lambda_{j}\right) / a_{k}, v_{j k}\right)$ as a Laurent series immediately gives the following generating function for the generalized Dedekind sums.

COROLlary 1. We have

$$
\begin{aligned}
\psi\left(S_{k}(x)\right) & =(-1)^{n} \sum_{\substack{r_{j}=0 \\
j \neq k}}^{\infty} s_{r_{0}, \ldots, r_{k-1}, r_{k+1}, \ldots, r_{n}} \frac{v_{0 k}^{r_{0}-1} \ldots v_{k-1, k}^{r_{k-1}-1} v_{k+1, k}^{r_{k+1}-1} \ldots v_{n k}^{r_{n}-1}}{r_{0} ! \ldots r_{k-1} ! r_{k+1} ! \ldots r_{n} !}
\end{aligned}
$$

where $s_{r_{0}, \ldots, r_{k-1}, r_{k+1}, \ldots, r_{n}}$ denotes

$$
\begin{array}{r}
s_{r_{0}, \ldots, r_{k-1}, r_{k+1}, \ldots, r_{n}}\left(a_{k} ; a_{0}, \ldots, a_{k-1}, a_{k+1}, \ldots, a_{n} ;\right. \\
\left.\lambda_{0}, \ldots, \lambda_{k-1}, \lambda_{k+1}, \ldots, \lambda_{n}\right) .
\end{array}
$$

In this generating function, each term is a quotient of homogeneous polynomials in the variables $X_{n}$. Each term thus has a well defined total degree, namely $r_{1}+\ldots+r_{k-1}+r_{k+1}+\ldots+r_{n}-n$. 
The reciprocity law comes from the following evaluation of the sum of the $\psi\left(S_{k}(x)\right)$.

THEOREM 2. We have

$$
\sum_{k=0}^{n} \psi\left(S_{k}(x)\right)= \begin{cases}0 & \text { if } n \text { is odd or } x \notin \mathbb{Z}^{n}, \\ 2^{-n} & \text { if } n \text { is even and } x \in \mathbb{Z}^{n} .\end{cases}
$$

Pro of. For each subset $B$ of $B_{0}=\{0,1, \ldots, n\}$ define

$$
S_{B}(x)=\sum_{y \in x+\mathbb{Z}^{n}} e^{y} \prod_{\substack{j=0 \\ j \notin B}}^{n} \omega\left(u_{j} \cdot y\right)
$$

so that in particular $S_{\{j\}}(x)=S_{j}(x)$. Also since each $a_{j}>0$ and $\sum_{j=0}^{n} a_{j} u_{j}$ $=0$ we can only have $u_{j} \cdot y \geq 0$ for all $j \in B_{0}$ if $y=0$. It follows that $S_{\emptyset}(x)=\delta / 2^{n+1}$ where

$$
\delta= \begin{cases}1 & \text { if } x \in \mathbb{Z}^{n} \\ 0 & \text { if } x \notin \mathbb{Z}^{n} .\end{cases}
$$

However if $i$ and $j$ are distinct elements of $B$, then $\omega\left(u_{k} \cdot\left(y+v_{i j}\right)\right)=\omega\left(u_{k} \cdot y\right)$ for each $k \notin B$ so that $\left(e^{v_{i j}}-1\right) S_{B}(x)=0$. Thus for $|B| \geq 2$ we have $\psi\left(S_{B}(x)\right)=0$.

We now consider the following sum:

$$
\Sigma=\sum_{y \in x+\mathbb{Z}^{n}} e^{y} \prod_{k=0}^{n}\left(1-\omega\left(u_{k} \cdot y\right)\right) .
$$

First of all $1-\omega(t)=\omega(-t)$ and so only the terms with $u_{k} \cdot y \leq 0$ for all $y$ can be zero. But this necessitates that $y=0$ and so $\Sigma=\delta / 2^{n+1}$. Expanding out the product gives

$$
\Sigma=\sum_{B \subseteq B_{0}}(-1)^{n+1-|B|} S_{B}(x) .
$$

Hence

$$
\psi(\Sigma)=(-1)^{n+1} S_{\emptyset}(x)+(-1)^{n} \sum_{k=1}^{n} \psi\left(S_{k}(x)\right) .
$$

Rearranging gives

$$
\sum_{k=0}^{n} \psi\left(S_{k}(x)\right)=\left(1+(-1)^{n}\right) \delta / 2^{n+1},
$$

which is equivalent to the stated identity.

Formal sums of the form $S_{k}(x)$ were used in [3] to construct cocycles of $\mathrm{PGL}_{2}(\mathbb{Q})$ and $\mathrm{PGL}_{3}(\mathbb{Q})$. 
5. Examples. We can read off reciprocity laws by equating the terms of a given degree on each side of the identity of Theorem 2 . We shall do this explicitly for the terms of degree zero. We first note that the Dedekind sums are essentially trivial unless all the parameters $r_{j}$ are strictly positive.

LEMMA 4. If some $r_{j}$ are 0 then

$$
s_{r_{1}, \ldots, r_{n}}\left(a ; b_{1}, \ldots, b_{n} ; \lambda_{1}, \ldots, \lambda_{n}\right)=a^{n-1-\left(r_{1}+\ldots+r_{n}\right)} \widetilde{B}_{r_{1}}\left(\lambda_{1}\right) \ldots \widetilde{B}_{r_{n}}\left(\lambda_{n}\right) .
$$

Proof. Assume without loss of generality that $r_{n}=0$. The condition that $a \mid \sum_{j} b_{j} i_{j}$ means that each choice of $i_{1}, \ldots, i_{n-1}$ determines a unique $i_{n}$. As $\widetilde{B}_{0}(t)=1$ we have

$$
\begin{aligned}
s_{r_{1}, \ldots, r_{n}}\left(a ; b_{1}, \ldots, b_{n} ; \lambda_{1}, \ldots, \lambda_{n}\right) & =\sum_{i_{1}, \ldots, i_{n-1}=0}^{a-1} \prod_{j=0}^{n-1} \widetilde{B}_{r_{j}}\left(\left(i_{j}+\lambda_{j}\right) / a\right) \\
& =\prod_{j=0}^{n-1} \sum_{i=0}^{a-1} \widetilde{B}_{r_{j}}\left(\left(i+\lambda_{j}\right) / a\right) \\
& =\prod_{j=0}^{n-1} a^{1-r_{j}} \widetilde{B}_{r_{j}}\left(\lambda_{j}\right)
\end{aligned}
$$

by the distribution relation. Since $\widetilde{B}_{r_{n}}\left(\lambda_{n}\right)=\widetilde{B}_{0}\left(\lambda_{n}\right)=1$ the lemma now follows.

We now consider the terms of degree zero in the generating function $\psi\left(S_{k}(x)\right)$. For convenience write

$$
s^{(k)}=s_{1,1, \ldots, 1}\left(a_{k} ; a_{0}, \ldots, a_{k-1}, a_{k+1}, \ldots, a_{n} ; \lambda_{0}, \ldots, \lambda_{k-1}, \lambda_{k+1}, \ldots, \lambda_{n}\right) .
$$

Let $R_{k}$ denote the set of $(n+1)$-tuples $r=\left(r_{0}, \ldots, r_{n}\right)$ of non-negative integers with $r_{0}+\ldots+r_{n}=n$ and $r_{k}=r_{j}=0$ for some $j \neq k$. Then by Lemma 4 the sum of the degree zero terms of $\psi\left(S_{k}(x)\right)$ is

$$
\Sigma_{k}=s^{(k)}+\sum_{r \in R_{k}} \frac{1}{a_{k}} \prod_{\substack{j=0 \\ j \neq k}}^{n} \widetilde{B}_{r_{j}}\left(\lambda_{j}\right) \frac{v_{j k}^{r_{j}-1}}{r_{j} !} .
$$

Let us write $v_{j k}=a_{j} a_{k} w_{j k}$. Then $w_{j k}+w_{k j}=0$ and $w_{i j}+w_{j k}+w_{k i}=0$ for all $i, j$ and $k$. We thus have $w_{j k}=x_{k}-x_{j}$ for some $x_{k}$, for instance if $x_{k}=w_{0 k}$ for $k>0$ and $x_{0}=0$. Thus

$$
\Sigma_{k}=s^{(k)}+\frac{1}{a_{0} \ldots a_{n}} \sum_{r \in R_{k}} \prod_{\substack{j=0 \\ j \neq k}}^{n} \frac{\widetilde{B}_{r_{j}}\left(\lambda_{j}\right)}{r_{j} !} a_{j}^{r_{j}}\left(x_{k}-x_{j}\right)^{r_{j}-1} .
$$


Let us put $R=\bigcup_{k=0}^{n} R_{k}$ and

$$
D_{k}=\prod_{\substack{j=0 \\ j \neq k}}^{n}\left(x_{k}-x_{j}\right)
$$

Then

$$
\sum_{k=0}^{n}\left(\Sigma_{k}-s^{(k)}\right)=\frac{1}{a_{0} \ldots a_{n}} \sum_{r \in R} \frac{\widetilde{B}_{r_{0}}\left(\lambda_{0}\right) \ldots \widetilde{B}_{r_{n}}\left(\lambda_{n}\right) a_{0}^{r_{0}} \ldots a_{n}^{r_{n}}}{r_{0} ! \ldots r_{n} !} T_{r}
$$

where

$$
T_{r}=\sum_{k=0}^{n} \frac{1}{D_{k}} \prod_{j=0}^{n}\left(x_{k}-x_{j}\right)^{r_{j}} .
$$

Lemma 5. Let $r=\left(r_{0}, r_{1}, \ldots, r_{n}\right)$ be an $n$-tuple of positive integers with $r_{0}+r_{1}+\ldots+r_{n}=n$. Then $T_{r}$, as defined above, equals 1 .

Proof. Define $f(x)=\prod_{j=0}^{n}\left(x-x_{j}\right)^{r_{j}}$. Then $f$ is a monic polynomial of degree $n$. Consider the determinant

$$
\Delta=\left|\begin{array}{cccc}
1 & 1 & \ldots & 1 \\
x_{0} & x_{1} & \ldots & x_{n} \\
x_{0}^{2} & x_{1}^{2} & \ldots & x_{n}^{2} \\
\vdots & \vdots & \ddots & \vdots \\
x_{0}^{n-1} & x_{1}^{n-1} & \ldots & x_{n}^{n-1} \\
f\left(x_{0}\right) & f\left(x_{1}\right) & \ldots & f\left(x_{n}\right)
\end{array}\right|
$$

By using elementary row operations we see that $\Delta$ equals the Vandermonde determinant

$$
\left|\begin{array}{cccc}
1 & 1 & \ldots & 1 \\
x_{0} & x_{1} & \ldots & x_{n} \\
x_{0}^{2} & x_{1}^{2} & \ldots & x_{n}^{2} \\
\vdots & \vdots & \ddots & \vdots \\
x_{0}^{n-1} & x_{1}^{n-1} & \ldots & x_{n}^{n-1} \\
x_{0}^{n} & x_{1}^{n} & \ldots & x_{n}^{n}
\end{array}\right|=\prod_{0 \leq i<j \leq n}\left(x_{j}-x_{i}\right) .
$$

But expanding out along the last row gives

$$
\Delta=\sum_{k=0}^{n} f\left(x_{k}\right)(-1)^{n-k} \prod_{\substack{0 \leq i<j \leq n \\ i \neq k \neq j}}\left(x_{j}-x_{i}\right) .
$$

Taking the quotient of these two expressions for $\Delta$ establishes the lemma. 
We thus get the reciprocity law: suppose that $\sum_{k=0}^{n} a_{k} \lambda_{k}=0$. Then

$$
\sum_{k=0}^{n} s^{(k)}=\frac{\delta\left(1+(-1)^{n}\right)}{2^{n+1}}-\frac{1}{a_{0} \ldots a_{n}} \sum_{r \in R} \prod_{j=0}^{n} \frac{\widetilde{B}_{r_{j}}\left(\lambda_{j}\right) a^{r_{j}}}{r_{j} !}
$$

where $R$ is the set of $(n+1)$-tuples $r=\left(r_{0}, \ldots, r_{n}\right)$ of non-negative integers summing to $n$ with at least two of the $r_{k}$ vanishing, and $\delta=1$ if all the $\lambda_{k}$ are in $\mathbb{Z}$ and $\delta=0$ otherwise.

By reading off the coefficients of terms of positive degree in the $\psi\left(S_{k}(x)\right)$ we can obtain further reciprocity laws; however they rapidly become cumbersome to write down.

In [2] $\mathrm{Hu}$ proved a reciprocity law akin to Theorem 2 by generalizing the method of Hall, Wilson and Zagier [1]. However he did not use this to deduce explicit reciprocity laws for the Dedekind sums themselves.

6. Acknowledgements. The work described in this paper was done when the author was a Visiting Fellow in the Division of Information and Communication Sciences at Macquarie University. He wishes to thank Alf van der Poorten for his kind invitation, David Solomon for awakening his interest in this subject and providing him with references, and Gerry Myerson and David Solomon for alerting him to errors in earlier versions of the paper.

\section{References}

[1] R. R. Hall, J. C. Wilson and D. Zagier, Reciprocity formulae for general DedekindRademacher sums, Acta Arith. 73 (1995), 389-396.

[2] S. Hu, Shintani cocycles and generalized Dedekind sums, Ph.D. thesis, Univ. of Pennsylvania, 1997.

[3] S. Hu and D. Solomon, Properties of higher-dimensional Shintani generating functions and cocycles on $\mathrm{PGL}_{3}(\mathbb{Q})$, Proc. London Math. Soc., to appear.

[4] H. Rademacher, Generalization of the reciprocity formula for Dedekind sums, Duke Math. J. 21 (1954), 391-397.

[5] D. Solomon, Algebraic properties of Shintani's generating functions: Dedekind sums and cocycles on $\mathrm{PGL}_{2}(\mathbb{Q})$, Compositio Math. 112 (1998), 333-362.

[6] D. Zagier, Higher dimensional Dedekind sums, Math. Ann. 202 (1973), 149-172.

Division of Information and Communication Sciences Macquarie University

Sydney, New South Wales 2109

Australia
Current address: School of Mathematical Sciences University of Exeter

Exeter

EX4 4QE, UK

E-mail: rjc@maths.ex.ac.uk 